\title{
The risk of venous thromboembolism in cancer patients receiving chemotherapy: a meta-analysis with systematic review
}

\author{
Yun Bao ${ }^{1,2}, \mathrm{Xu} \mathrm{Wan}^{3}, \mathrm{Jie} \mathrm{Fu}^{3}, \mathrm{Bin} \mathrm{Wu}^{3}$ \\ ${ }^{1}$ Institute of Clinical Research and Evidence Based Medicine, Gansu Provincial Hospital, Lanzhou, China; ${ }^{2}$ Department of Pharmacy, Gansu \\ Provincial Hospital, Lanzhou, China; ${ }^{3}$ Department of Pharmacy, Ren Ji Hospital, South Campus, School of Medicine, Shanghai Jiao Tong \\ University, Shanghai, China \\ Contributions: (I) Conception and design: B Wu; (II) Administrative support: None; (III) Provision of study materials or patients: B Wu; (IV) \\ Collection and assembly of data: Y Bao, B Wu, J Fu; (V) Data analysis and interpretation: Y Bao, B Wu; (VI) Manuscript writing: All authors; (VII) \\ Final approval of manuscript: All authors. \\ Correspondence to: Dr. Bin Wu. Medical Decision and Economic Group, Department of Pharmacy, Ren Ji Hospital, South Campus, School of \\ Medicine, Shanghai Jiao Tong University, Jiangyue Road 2000, Shanghai, China. Email: scilwsjtu-wb@yahoo.com; Yun Bao. Institute of Clinical \\ Research and Evidence Based Medicine, Gansu Provincial Hospital, Lanzhou, China. Email: baoyun2009@126.com.
}

\begin{abstract}
Background: The Khorana score was developed to predict the risk of venous thromboembolism (VTE) in cancer patients receiving chemotherapy. However, the utility of the Khorana score remains controversial since different studies report varying results. This meta-analysis aims to analyze the incidence of VTE with different risk stratifications using the Khorana score for overall follow-up time, incidence of deepvein thrombosis (DVT), incidence of pulmonary embolism (PE) and bleeding in cancer patients receiving chemotherapy.
\end{abstract}

Methods: A systemic search was performed using PubMed, Embase, Cochrane Library and Web of Science for studies describing VTE incidence in cancer patients undergoing chemotherapy. The incidence of VTE was calculated using R computing software.

Results: We included 13 studies in this meta-analysis, with a total of 5,852 cancer patients and 424 VTE cases. Results revealed that overall incidence of low, intermediate and high-risk groups were $2 \%$ (95\% CI: 1-6\%), 11\% (95\% CI: 6-18\%) and 14\% (95\% CI: 9-20\%), respectively. The overall incidence of DVT and PE were 6\% (95\% CI: 4-10\%) and 4\% (95\% CI: 2-7\%), respectively. Lastly, bleeding rate was 4\% (95\% CI: $2-8 \%)$.

Conclusions: According to this meta-analysis, the Khorana score is suitable for cancer patients receiving chemotherapy in a 3-6-month timeframe rather than "forever". The incidence of PE in this population was significantly greater than what was observed for non-cancer patients. More than half of VTE events occurred within 6 months of commencing chemotherapy.

Keywords: Venous thromboembolism (VTE); cancer; chemotherapy; Khorana score; meta-analysis

Submitted Apr 13, 2020. Accepted for publication Nov 26, 2020.

doi: $10.21037 /$ atm-20-3292

View this article at: http://dx.doi.org/10.21037/atm-20-3292

\section{Introduction}

Venous thromboembolisms (VTE), such as deep-vein thrombosis (DVT) and pulmonary embolism (PE), are major complications that lead to death in cancer patients (1). There are many factors influencing the increased risk of VTE in cancer patients, including chemotherapy. Due to direct effects on vascular endothelium (2), there is a 6- to 7-fold risk of cancer-associated VTE observed in patients treated with chemotherapy (3). Regardless, few cancer patients receive thromboprophylaxis during chemotherapy (4). Presently, clinical guidelines do not clearly illustrate the conditions where patients should receive primary 
VTE prevention during chemotherapy (5). Therefore, it is necessary to monitor for VTE in cancer patients undergoing chemotherapy.

The Khorana score, or the best validated model, has been recommended by the National Comprehensive Cancer Network (NCCN) Clinical Practice Guidelines in Oncology to identify cancer patients at risk for VTE and those who would be eligible for thromboprophylaxis (6). By applying five risk factors, the Khorana score (Table S1) classifies cancer patients based on three risk stratifications, including low (score $=0$ ), intermediate (score $=1-2$ ) and high ( score $\geq 3$ ) risk groups. However, previous studies have reported that the incidence of VTE ranges, and these rates are $0.8-13 \%, 1.8-$ $15.9 \%$ and $6.7-41.4 \%$ in the low, intermediate and highrisk groups, respectively (7). Moreover, other studies have indicated no differences between intermediate and highrisk groups in relation to VTE incidence $(8,9)$, even though there is a higher rate observed in intermediate compared to high-risk groups (10). Recently, two randomized controlled trials (RCTs) $(11,12)$ considered cancer patients with a score of 2 points or greater at high risk for developing VTE. By pooling published data, this meta-analysis aims to investigate the incidence of VTE in cancer patients undergoing chemotherapy with different risks stratified using the Khorana score. This article is presented in accordance with the PRISMA reporting checklist (available at http://dx.doi. org/10.21037/atm-20-3292).

\section{Methods}

\section{Search strategy}

Meta-analysis was performed based on the Preferred Reporting Items for Systematic Reviews and Meta-analysis (PRISRMA) guidelines. Two researchers independently searched the literature. Databases used for eligible studies included PubMed, Embase, Cochrane Library, and Web of Science, from January 1, 2008 to May 1, 2019. Conflicts between the two researchers were resolved through discussion. To retrieve all eligible articles, search terms ((Khorana or KRS) and (neoplasms OR neoplas* OR cancer OR malign* OR tumor*)) were used. There were no limits placed on the study design or language. Studies published before 2008 were not included since the Khorana score was released in 2008. The CRD registration number is CRD42019135938.

\section{Inclusion and exclusion criteria}

Patient and study inclusion criteria included: (I) adults (>18 years old) diagnosed with a malignancy and undergoing chemotherapy; (II) no prior history of VTE and not receiving thromboprophylaxis at baseline; (III) risk stratification of VTE classified using the Khorana score, calculated on the first day of chemotherapy or the day before chemotherapy; (IV) RCTs, retrospective cohort studies or prospective cohort studies. Patient and study exclusion criteria included studies containing recurrent VTE, patients who were pregnant and individuals receiving thromboprophylaxis.

\section{Outcomes}

Outcomes included incidence of all VTE and bleeding events. VTE was defined as symptomatic or asymptomatic distal lower-extremity DVT, upper-extremity DVT, proximal lower-extremity DVT, upper-extremity DVT, superficial vein thrombosis, $\mathrm{PE}$ and splanchnic vein thrombosis. Bleeding events are composed of major bleeding and clinically relevant non-major bleeding. Major bleeding was defined by the International Society as Thrombosis and Hemostasis.

\section{Data extraction}

The following data were extracted: study design, publication year, study location, mean age, sex, proportion (male), chemotherapy regimen, follow-up time, risk category, outcomes, frequency of VTE and bleeding in different stratifications.

\section{Quality assessment}

In this meta-analysis, the quality of studies was assessed using a customized Newcastle-Ottawa Scale (NOS) quality assessment scale analyzing study representativeness, applicability of the Khorana score, outcome measurement, adequacy of cohort follow-up and applicability of outcomes (Table S2).

\section{Statistical methods}

All data were analyzed using $\mathrm{R}$ computing software, version (3.6.0) (R Foundation for Statistical Computing). The meta-analysis was analyzed using logit transformation (13). The I-squared $\left(\mathrm{I}^{2}\right)$ statistic was calculated to quantify 


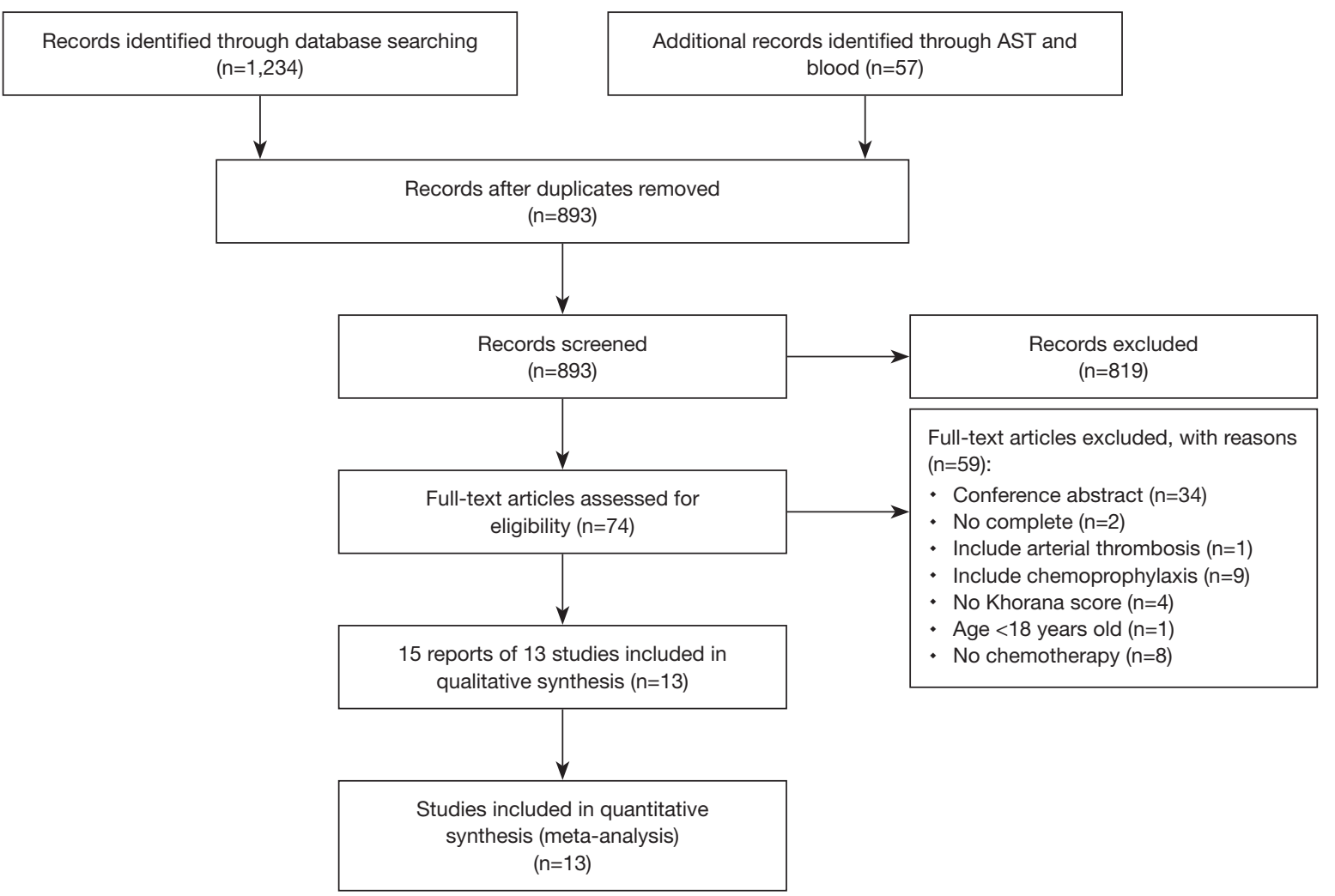

Figure 1 Flowchart illustrating the search strategy used for study inclusion.

heterogeneity among the studies. The meta-analysis was performed using a random-effects model and a $\mathrm{I}^{2} \geq 75 \%$ was considered as high heterogeneity. For overall metaanalysis, data were used belonging to the median followup time and VTE incidence was analyzed as well as the corresponding $95 \%$ confidence interval (95\% CI) for three risk stratifications. Stratified analyses were conducted based on the follow-up period. Publication bias was investigated using the Egger test (14). Sensitivity analysis was performed by excluding studies with high bias.

\section{Results}

\section{Literature search}

A total of 1,234 articles were obtained from all databases using custom searches. After deleting duplicates, 893 records were identified and 74 studies were selected for fulltext review after reviewing the titles and abstracts of the records. The 59 remaining reports were excluded. There were 15 reports from the 13 studies meeting the inclusion criteria that were included in the meta-analysis (Figure 1).

\section{Study characteristics}

Characteristics of the 13 studies included in the meta-analysis are shown in Table 1. There was a total of 5,852 cancer patients and 424 diagnosed with VTE. Low-risk stratification was observed in five studies and there were a total of 1,300 cancer patients and 54 VTE cases. There were 9 studies including 3,029 cancer patients with intermediate risk and 230 patients diagnosed with VTE. There were 11 studies involving 850 cancer patients with a high risk and 104 VTE cases. This study represented all cancer types, including esophageal, gastric, pancreatic, lung, lymphoma, bladder and breast cancers. Follow-up time frames ranged from 2.5 to 24 months and the mean age ranged from 50 to 66 years. A study performed by Rupa-Matysek et al. (2017) (19) contained four datasets exhibiting different follow-up periods. The study performed by van Es et al. (2017) (9) contained three datasets. To estimate overall DVT, PE and bleeding rate, two additional reports were included $(12,25)$. Most studies were identified as high quality and just 1 study showed high bias. The method used to analyze the included studies is shown in Table 2. 


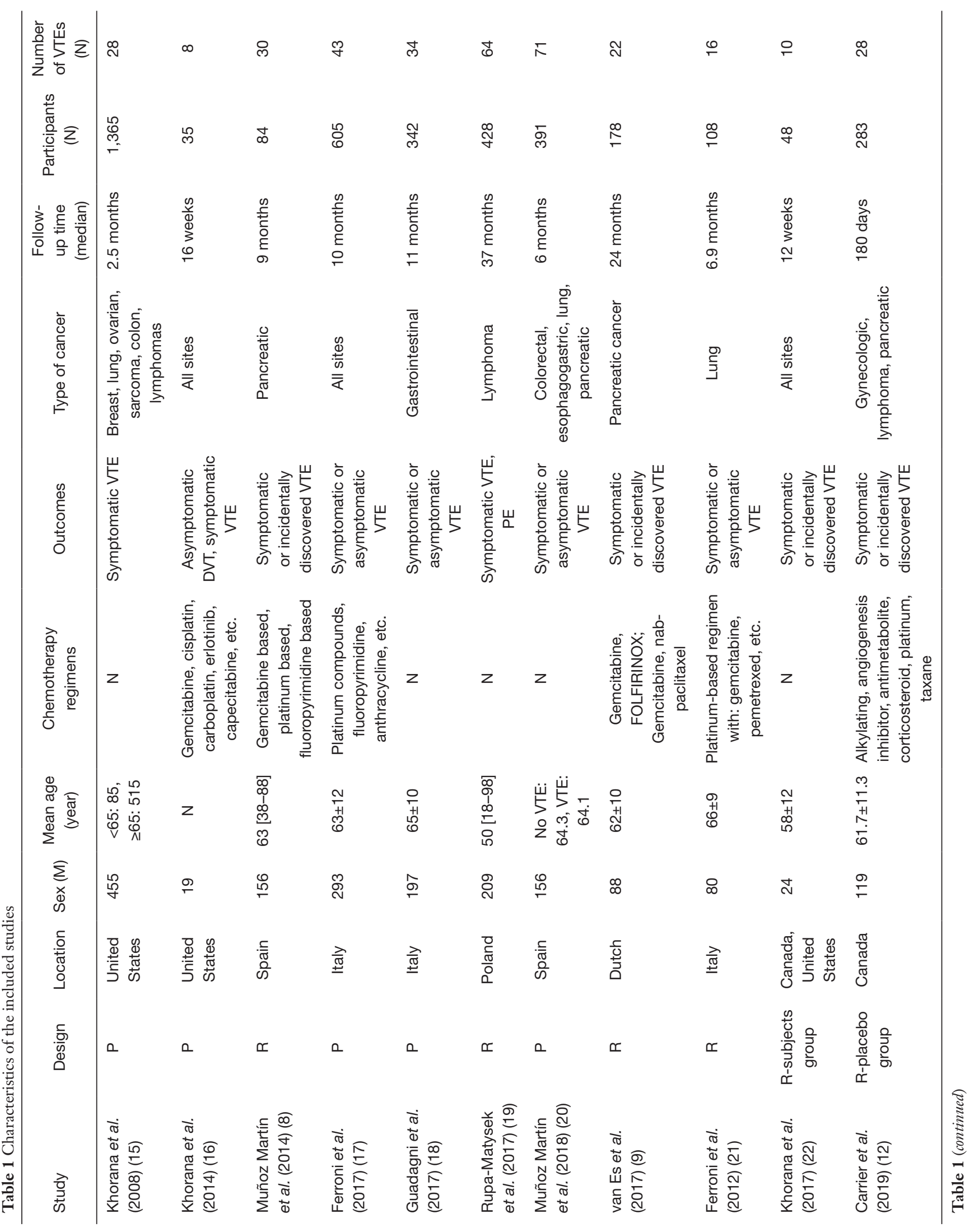




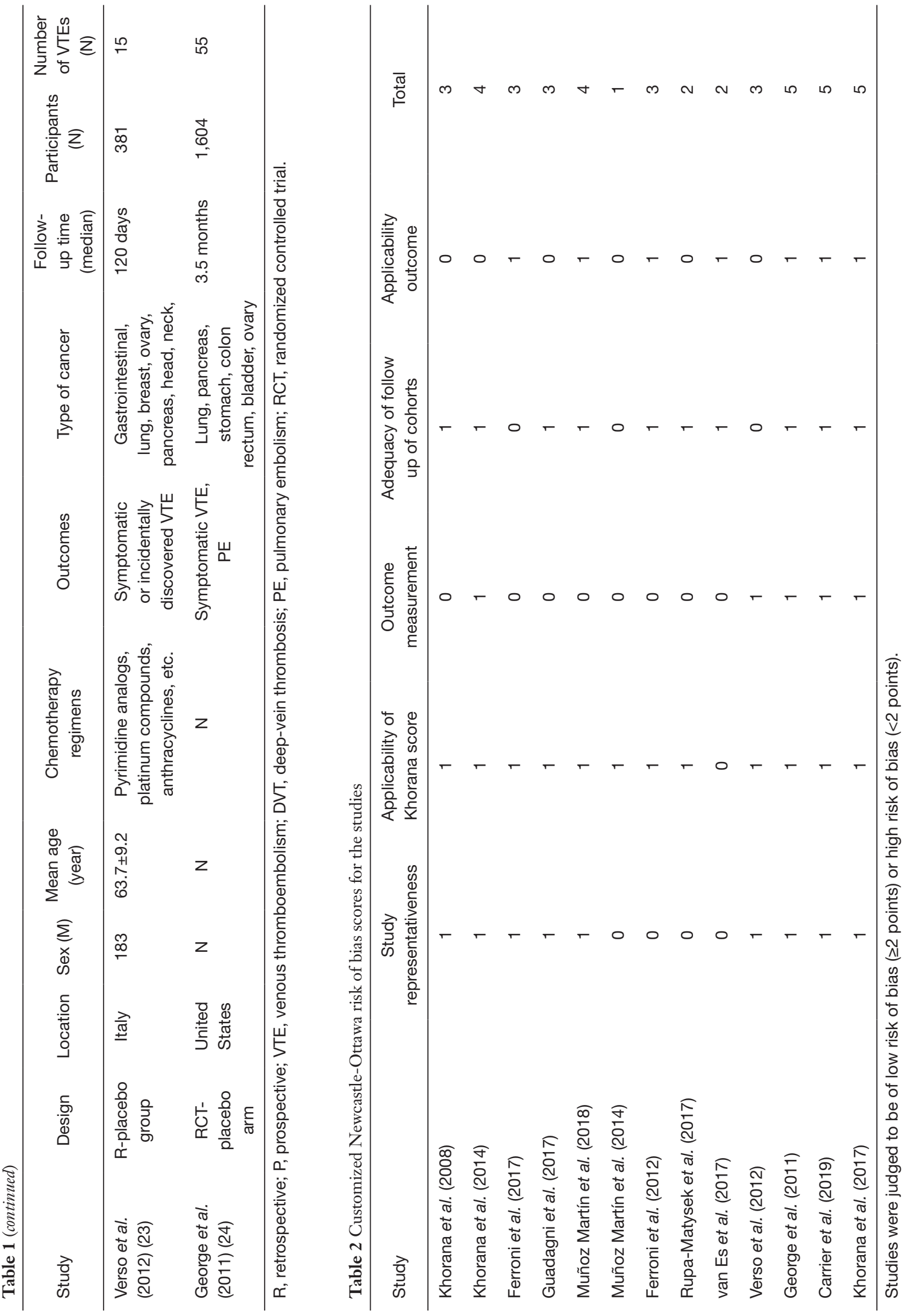




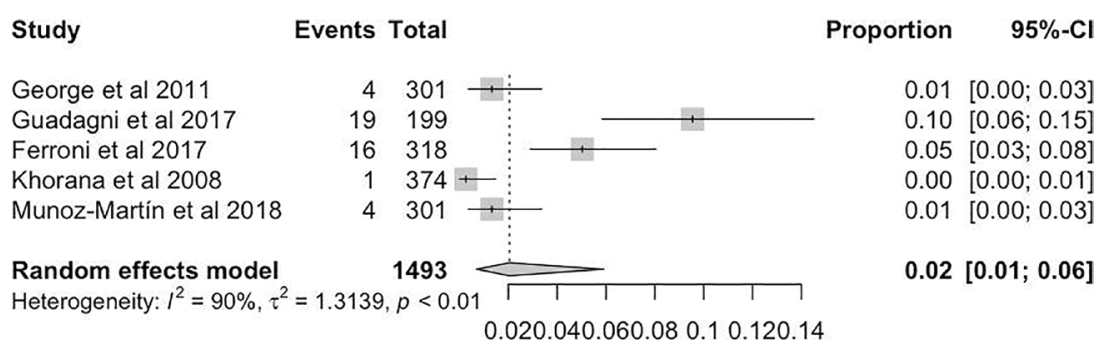

Figure 2 Forest plot of the pooled overall VTE incidence in cancer patients at low risk. VTE, venous thromboembolism.

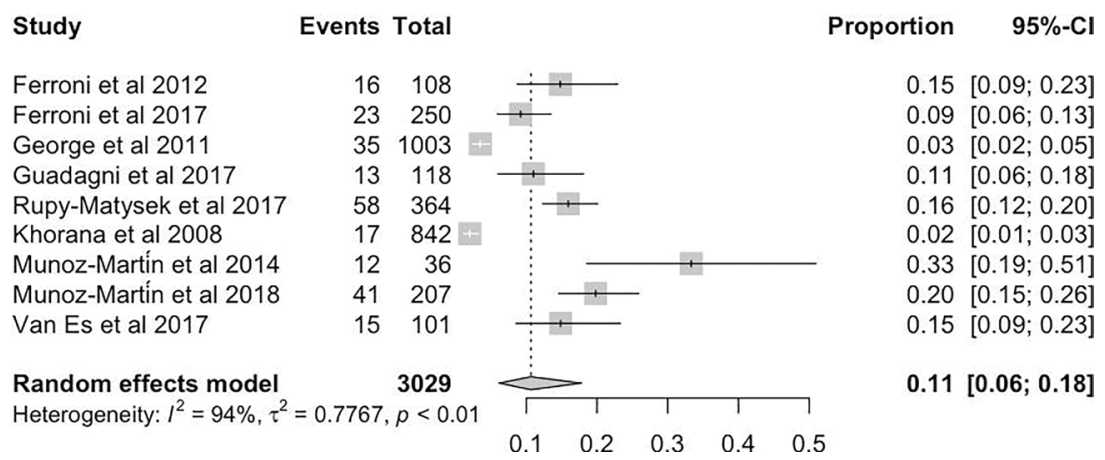

Figure 3 Forest plot of the pooled overall VTE incidence in cancer patients at intermediate risk. VTE, venous thromboembolism.

\section{Overall VTE incidence at different risk scores}

The incidence of VTE was $2 \%$ (95\% CI: $1-6 \%, \mathrm{I}^{2}=90 \%$, $\mathrm{P}<0.01), 11 \%\left(95 \%\right.$ CI: $\left.6-18 \%, \mathrm{I}^{2}=94 \%, \mathrm{P}<0.01\right)$ and 14\% (95\% CI: 9-20\%, $\left.\mathrm{I}^{2}=75 \%, \mathrm{P}<0.01\right)$ in cancer patients with low-risk [Khorana Risk Score (KRS) =0] (Figure 2), intermediate-risk (KRS =1-2) (Figure 3) and high-risk scores (KRS $\geq 3$ ) (Figure 4), respectively. When considering two or more points as high-risk instead of three or more points, the incidence of VTE in cancer patients with high risk was $14 \%$ (95\% CI: $\left.8-23 \%, \mathrm{I}^{2}=79 \%, \mathrm{P}<0.01\right)$ (Figure 5).

\section{VTE incidence at different follow-up times}

The incidence of cancer-related VTE differed based on follow-up times (8). Khorana et al. (2013) (26) reported that $66-72.5 \%$ of VTE events occurred within 6 months. In this meta-analysis, the median time of the first VTE event was $1.3,2.5,3.2$ and 4.7 months as shown by four different studies (16-19). According to the study reported by van Es et al. (2017) (9), there was no difference in VTE incidence between 12 and 24 months. Therefore, we classified the studies into two groups based on follow-up time for analysis. The two groups were divided into 3-6 and 7-12 months.
The incidence of VTE at 3-6 and 7-12 months was $1 \%$ (95\% CI: $0-2 \%, \mathrm{I}^{2}=1 \%, \mathrm{P}=0.32$ ) and 7\% (95\% CI: 4-11\%, $\left.\mathrm{I}^{2}=48 \%, \mathrm{P}=0.05\right)$ at low risk, $7 \%\left(95 \% \mathrm{CI}: 3-14 \%, \mathrm{I}^{2}=95 \%\right.$, $\mathrm{P}<0.01)$ and $14 \%\left(95 \% \mathrm{CI}: 11-19 \%, \mathrm{I}^{2}=63 \%, \mathrm{P}<0.01\right)$ at intermediate risk and $12 \%$ (95\% CI: $8-17 \%, \mathrm{I}^{2}=69 \%$, $\mathrm{P}<0.01)$ and $15 \%\left(95 \%\right.$ CI: $\left.9-26 \%, \mathrm{I}^{2}=66 \%, \mathrm{P}<0.01\right)$ at high risk, respectively (Figure 6, Figures S1-S3).

\section{DVT, PE and bleeding incidence rates}

The incidence of DVT and PE in the cases analyzed was $6 \%\left(95 \%\right.$ CI: $\left.4-10 \%, \mathrm{I}^{2}=86 \%, \mathrm{P}<0.01\right)$ and $4 \%(95 \%$ CI: $2-7 \%, \mathrm{I}^{2}=85 \%, \mathrm{P}<0.01$ ), respectively (Figures 7,8 ). Bleeding incidence was $4 \%$ (95\% CI: $2-8 \%, \mathrm{I}^{2}=81 \%$, $\mathrm{P}<0.01$ ) (Figure 9).

\section{Sensitivity analysis and publication bias}

There was no significant publication bias detected by Egger's test (low risk: $\mathrm{P}=0.0992$, intermediate risk: $\mathrm{P}=0.9143$, high risk: $\mathrm{P}=0.7999)$. To assess whether high bias affected heterogeneity, the study performed by Muñoz Martín et al. (2014) was removed (8) and the overall incidence of VTE was 9\% (95\% CI: $\left.5-15 \%, \mathrm{I}^{2}=94 \%, \mathrm{P}<0.01\right)$ and $14 \%$ (95\% CI: 


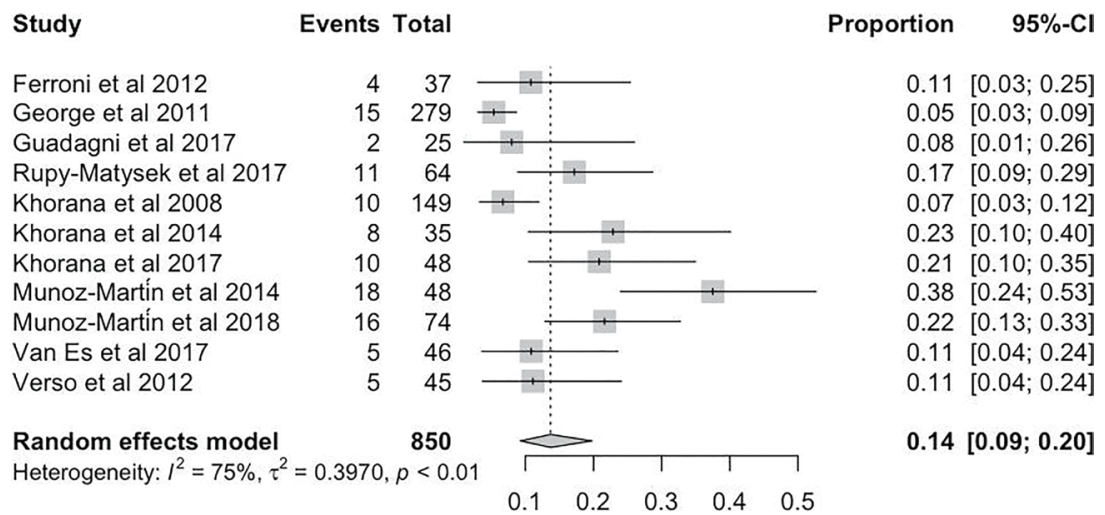

Figure 4 Forest plot of the pooled overall VTE incidence in cancer patients at high risk. VTE, venous thromboembolism.

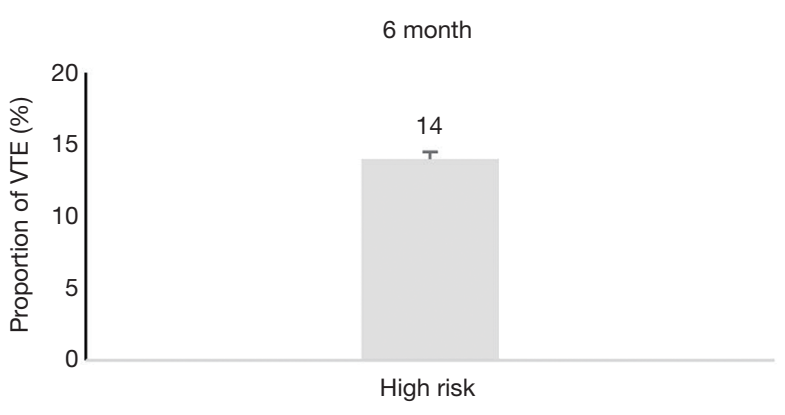

Figure 5 The VTE incidence in cancer patients when considering two points or more as high risk. VTE, venous thromboembolism.

9-20\%, $\left.\mathrm{I}^{2}=75 \%, \mathrm{P}<0.01\right)$ at intermediate and high-risk levels, respectively. The incidence of VTE at 3-6 and 7-12 months was $7 \%$ (95\% CI: $\left.3-14 \%, \mathrm{I}^{2}=95 \%, \mathrm{P}<0.01\right)$ and $13 \%(95 \%$ CI: $\left.10-16 \%, \mathrm{I}^{2}=26 \%, \mathrm{P}=0.22\right)$ at intermediate risk and $12 \%\left(95 \%\right.$ CI: $\left.8-17 \%, \mathrm{I}^{2}=69 \%, \mathrm{P}<0.01\right)$ and $12 \%(95 \% \mathrm{CI}$ : $\left.8-18 \%, \mathrm{I}^{2}=0 \%, \mathrm{P}=0.75\right)$ at high risk, respectively.

\section{Discussion}

Here, we systematically analyzed the incidence of VTE at different risk stratification levels using the Khorana score and overall bleeding rate in cancer patients undergoing chemotherapy. We demonstrated that the time frame Khorana score prediction was 3-6 months rather than "forever".

According to the meta-analysis, the incidence of DVT was higher than $\mathrm{PE}$, which was significantly greater than the rate in non-cancer patients $(0.2 \%)$ (26). Two studies $(8,26)$ reported that $\mathrm{PE}$, which has a high fatality rate, was asymptomatic and combined with DVT in most cases.
Meanwhile, the guidelines recommend anticoagulant treatment for incidental VTE events as for cancer patients with symptomatic VTE (27).

Our analysis revealed a higher incidence of VTE than the study that developed the Khorana score since, besides one other study (19), the studies contained in our metaanalysis included both symptomatic and asymptomatic VTE as outcomes. However, the original study only included symptomatic VTE cases. Our analysis also indicated that over half of VTE events occurred in 6 months, similar to two other studies $(21,26)$. Interestingly, although the incidence of VTE differed at 6 months for different risk stratifications based on the Khorana score, the incidence of VTE at 12 months between intermediate and high-risk groups were the same. This result is similar to meta-analysis results published by Mulder et al. (28) that revealed that the Khorana score had limited use in ruling out future venous thromboembolic events ( $>6$ months) since it calculated laboratory data before chemotherapy. Recent RCTs $(11,12)$ and Mulder et al. (28) considered two or more points as high risk instead of 3 or more points. Our study agrees with this view by finding that VTE incidence was similar in cancer patients undergoing chemotherapy despite considering two to three or more points as high risk.

Additionally, the meta-analysis presented here confirmed the viewpoint of Imberti and colleagues (29) who showed that the incidence rate of cancer-related VTE in the real world is greater than the incidence rate of VTE in RCTs. A study conducted by van Es et al. (30) reported a 6-month VTE incidence based on a $6.4 \%$ Khorana score in low-tointermediate risk patients and a score of $9.8 \%$ in high-risk patients. However, in the study presented here, we observed higher incidence. This discrepancy may be partially 


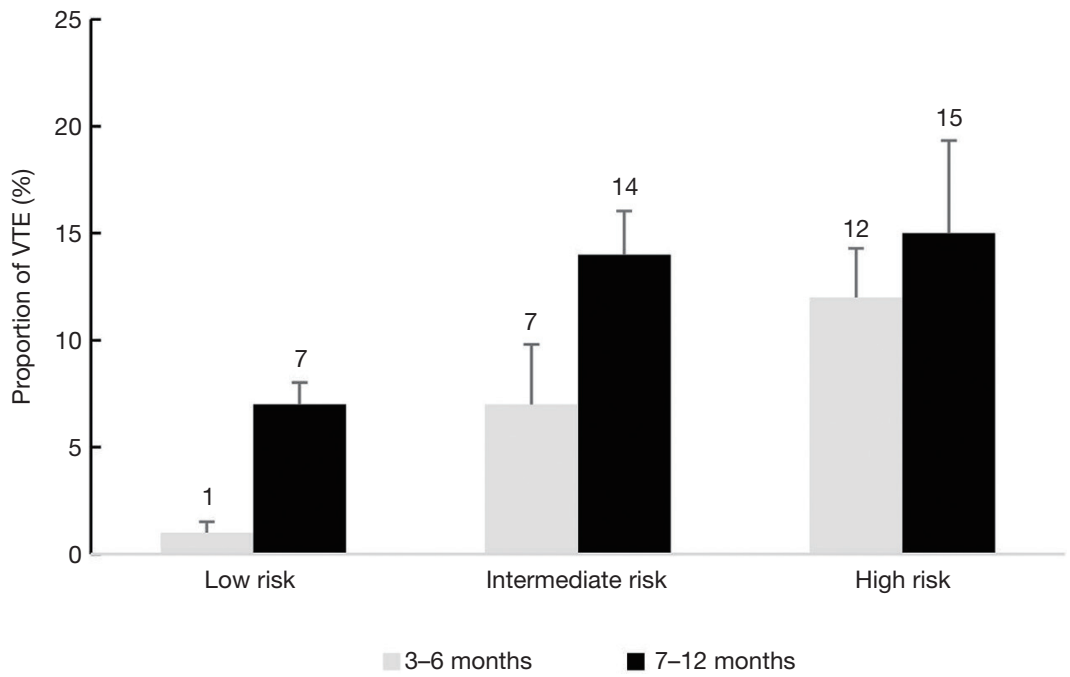

Figure 6 The incidence of different follow-up times in cancer patients at different risk levels. VTE, venous thromboembolism.

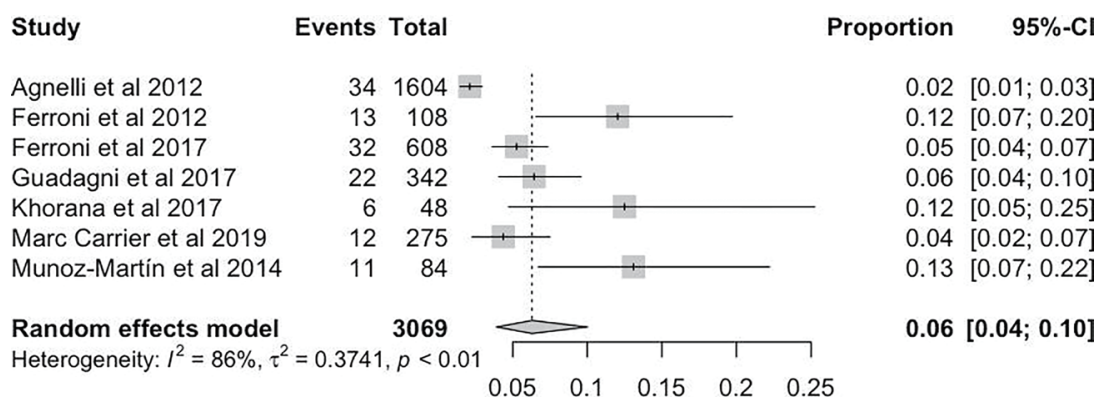

Figure 7 Forest plot of the pooled overall DVT incidence in cancer patients. DVT, deep-vein thrombosis.

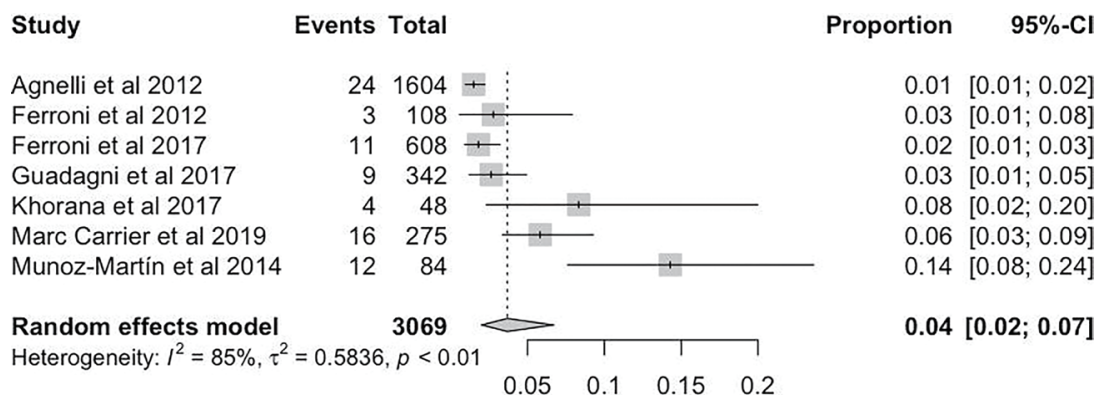

Figure 8 Forest plot of the pooled overall PE incidence in cancer patients. PE, pulmonary embolism.

explained by the fact that the study performed by van Es and colleagues only included RCTs and solid tumors (30). Meanwhile, observation cohort studies are more reliable in terms of demonstrating causality compared to other observational studies. The study performed by Mulder et al. (28), which included RCTs and cohort studies, showed that the incidence of VTE ranged from $5.1-11 \%$ in the first 6 months, which was lower than what was observed in our study. This difference may be caused by different anticancer therapies and thromboprophylaxis that the Mulder study did not exclude, where only some cancer patients received chemotherapy or showed thromboprophylaxis at 


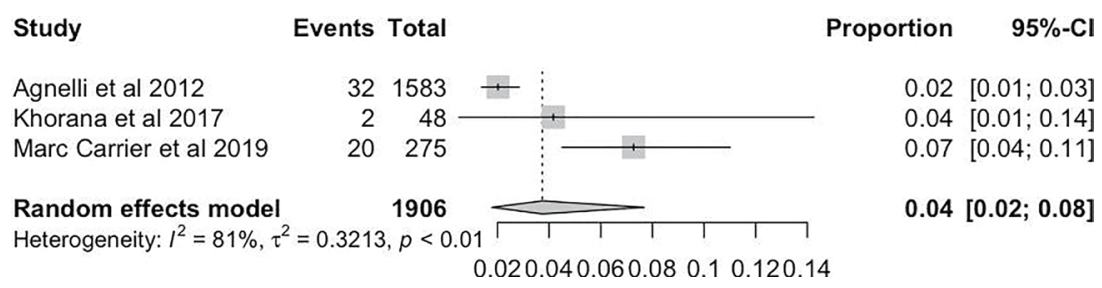

Figure 9 Forest plot of the pooled overall bleeding incidence in cancer patients.

baseline. It is worth noting that the incidence of cancerrelated VTE in the real world is higher than shown in the meta-analysis results. It is difficult to estimate the "true" rate because not all studies screen at baseline, which detects asymptomatic VTE. In the eligible studies included in our analyses, only the CASSINI trial (11), study by Khorana et al. (2014) (16) and study by Khorana et al. (2017) (22) contained baseline screening and screening during trials.

Interestingly, our results were contrary to recent cohort studies reporting that the Khorana scoring system is not ideal for predicting VTE regarding three aspects. First, two cohort studies $(19,31)$ reported that age ( $>60$ years), tumor burden, inflammatory activity and poor performance were independent risk factors for VTE but that the Khorana score was not since body mass index (BMI) and leukocyte count (WBC) were useless for assessing lymphoma progression. Thus, the Khorana score is not suitable for all malignancy types, particularly lymphoid malignancies. Second, some studies involved patients with lung and pancreatic cancers, which have a high incidence rate of VTE and failed to show the utility of the Khorana score. A meta-analysis conducted in 2017 also found that the Khorana score poorly differentiated between individuals at high and low risk for VTE in lung cancer patients (30), even though patients with stage IV lung cancer may benefit from extended prophylaxis due to a significant reduction in VTE and no increase in bleeding events (32). An individual, patient data meta-analysis performed in 2020 indicated that the Khorana score was unable to stratify lung cancer patients based on their VTE risk (33). Third, one study (17) including all cancer types also indicated that the Khorana score was not ideal for predicting cancer-associated VTE, possibly due to different treatment regimens. These differences emphasize that although the Khorana score is able to distinguish patients with various cancers at different risk stratification levels, it may not accurately perform when it comes to predicting VTE incidence in lung, pancreatic and lymphoid cancers.

Despite our findings, the study presented here also has some limitations. First, the study population involved patients that were heterogeneous in nature. Our meta-analysis evaluated overall VTE incidence with high heterogeneity, which was acceptable after subgroup analysis with different follow-up periods. However, there was insufficient subgroup analysis based on different cancer sites and chemotherapy regimens, which is significantly related to VTE incidence $(20,34)$. We also analyzed VTE incidence using a retrospective cohort, prospective cohort and RCT. However, this was not able to reduce heterogeneity (data not shown). Secondly, there was considerable bias risk in the enrolled studies. For example, studies included in our analysis involved various outcomes, which may affect the results. Finally, all included studies were conducted using Western populations. As a result, the work presented here may be subject to smallstudy effect bias in Eastern populations.

In conclusion, the meta-analysis presented here validated the utility of the Khorana score in cancer patients receiving chemotherapy in a 3-6-month time frame and suggests this may not be sufficient to distinguish VTE incidence between intermediate and high-risk groups after 6 months. However, there were some limitations associated with the cancer site and chemotherapy regimen. The incidence of $\mathrm{PE}$ in cancer patients was significantly greater than what was observed in non-cancer patients and more than half of VTE events occurred in 6 months. To establish a more reliable result between Khorana score and incidence of VTE in cancer patients, additional studies need to adjust for confounding factors and focus on the cancer site and chemotherapy regimen.

\section{Acknowledgments}

Funding: This work was sponsored by unrestricted grants from the Gansu Provincial Hospital's Youth Project (No. 20GSSY4-42), Natural Science Foundation of Gansu Province (No. 20JR10RA394), National Natural Science Foundation of China (No. 7172810 and 71373160). The funders had no role in the study design, data collection and 
analysis, decision to publish, or manuscript preparation.

\section{Footnote}

Reporting Checklist: The authors have completed the PRISMA reporting checklist. Available at http://dx.doi. org/10.21037/atm-20-3292

Conflicts of Interest: All authors have completed the ICMJE uniform disclosure form (available at http://dx.doi. org/10.21037/atm-20-3292). The authors have no conflicts of interest to declare.

Ethical Statement: The authors are accountable for all aspects of the work in ensuring that questions related to the accuracy or integrity of any part of the work are appropriately investigated and resolved.

Open Access Statement: This is an Open Access article distributed in accordance with the Creative Commons Attribution-NonCommercial-NoDerivs 4.0 International License (CC BY-NC-ND 4.0), which permits the noncommercial replication and distribution of the article with the strict proviso that no changes or edits are made and the original work is properly cited (including links to both the formal publication through the relevant DOI and the license). See: https://creativecommons.org/licenses/by-nc-nd/4.0/.

\section{References}

1. Khorana AA, Francis CW, Culakova E, et al. Thromboembolism is a leading cause of death in cancer patients receiving outpatient chemotherapy. J Thromb Haemost 2007;5:632-4.

2. Zangari M, Fink LM, Elice F, et al. Thrombotic events in patients with cancer receiving antiangiogenesis agents. J Clin Oncol 2009;27:4865-73.

3. Lyman GH, Khorana AA, Falanga A, et al. American Society of Clinical Oncology guideline: recommendations for venous thromboembolism prophylaxis and treatment in patients with cancer. J Clin Oncol 2007;25:5490-505.

4. Parker A, Peterson EA, Lee AYY, et al. Risk Stratification for the Development of Venous Thromboembolism in Hospitalized Patients with Cancer. Blood 2016;128:4728.

5. Khorana AA, Otten HM, Zwicker JI, et al. Prevention of venous thromboembolism in cancer outpatients: guidance from the SSC of the ISTH. J Thromb Haemost 2014;12:1928-31.
6. NCCN. NCCN clinical practice guidelines in Oncology: Cancer-Associated Venous Thromboembolic Disease. Available online: https://www.nccn.org/, 2018. V1.

7. Khorana AA. Cancer and coagulation. Am J Hematol 2012;87 Suppl 1:S82-7.

8. Muñoz Martín AJ, García Alfonso P, Rupérez Blanco $\mathrm{AB}$, et al. Incidence of venous thromboembolism (VTE) in ambulatory pancreatic cancer patients receiving chemotherapy and analysis of Khorana's predictive model. Clin Transl Oncol 2014;16:927-30.

9. van Es N, Franke VF, Middeldorp S, et al. The Khorana score for the prediction of venous thromboembolism in patients with pancreatic cancer. Thromb Res 2017;150:30-2.

10. Raskob GE, Angchaisuksiri P, Blanco AN, et al. Thrombosis: a major contributor to global disease burden. Arterioscler Thromb Vasc Biol 2014;34:2363-71.

11. Khorana AA, Soff GA, Kakkar AK, et al. Rivaroxaban for thromboprophylaxis in high-risk ambulatory patients with cancer. N Engl J Med 2019;380:720-8.

12. Carrier M, Abou-Nassar K, Mallick R, et al. Apixaban to prevent venous thromboembolism in patients with cancer. N Engl J Med 2019;380:711-9.

13. Singh A, Hussain S, Najmi AK. Number of studies, heterogeneity, generalisability, and the choice of method for meta-analysis. Journal of the Neurological Sciences 2017;381:347.

14. Egger M, Smith GD, Schneider M, et al. Bias in metaanalysis detected by a simple, graphical test. BMJ 1997;315:629-34.

15. Khorana AA, Kuderer NM, Culakova E, et al. Development and validation of a predictive model for chemotherapy-associated thrombosis. Blood 2008;111:4902-7.

16. Khorana AA, Rubens D, Francis CW. Screening high-risk cancer patients for VTE: a prospective observational study. Thromb Res 2014;134:1205-7.

17. Ferroni P, Zanzotto FM, Scarpato N, et al. Validation of a Machine Learning Approach for Venous Thromboembolism Risk Prediction in Oncology. Dis Markers 2017;2017:8781379.

18. Guadagni F, Riondino S, Formica V, et al. Clinical significance of glycemic parameters on venous thromboembolism risk prediction in gastrointestinal cancer. World J Gastroenterol 2017;23:5187-95.

19. Rupa-Matysek J, Gil L, Kazmierczak M, et al. Prediction of venous thromboembolism in newly diagnosed patients treated for lymphoid malignancies: validation of the 
Khorana Risk Score. Med Oncol 2017;35:5.

20. Muñoz Martín AJ, Ortega I, Font C, et al. Multivariable clinical-genetic risk model for predicting venous thromboembolic events in patients with cancer. $\mathrm{Br} \mathrm{J}$ Cancer 2018;118:1056-61.

21. Ferroni P, Martini F, Portarena I, et al. Novel highsensitive D-dimer determination predicts chemotherapyassociated venous thromboembolism in intermediate risk lung cancer patients. Clin Lung Cancer 2012;13:482-7.

22. Khorana AA, Francis CW, Kuderer NM, et al. Dalteparin thromboprophylaxis in cancer patients at high risk for venous thromboembolism: A randomized trial. Thromb Res 2017;151:89-95.

23. Verso M, Agnelli G, Barni S, et al. A modified Khorana risk assessment score for venous thromboembolism in cancer patients receiving chemotherapy: the Protecht score. Intern Emerg Med 2012;7:291-2.

24. George D, Agnelli G, Fisher W, et al. Venous Thromboembolism (VTE) Prevention with Semuloparin in Cancer Patients Initiating Chemotherapy: BenefitRisk Assessment by VTE Risk in SAVE-ONCO. Blood 2011;118:206.

25. Agnelli G, George DJ, Kakkar AK, et al. Semuloparin for thromboprophylaxis in patients receiving chemotherapy for cancer. N Engl J Med 2012;366:601-9.

26. Khorana AA, Dalal M, Lin J, et al. Incidence and predictors of venous thromboembolism (VTE) among ambulatory high-risk cancer patients undergoing chemotherapy in the United States. Cancer 2013;119:648-55.

27. Lyman GH, Bohlke K, Khorana AA, et al. Venous thromboembolism prophylaxis and treatment in patients

Cite this article as: $\mathrm{Bao} \mathrm{Y}, \mathrm{Wan} \mathrm{X}, \mathrm{Fu} \mathrm{J}, \mathrm{Wu}$ B. The risk of venous thromboembolism in cancer patients receiving chemotherapy: a meta-analysis with systematic review. Ann Transl Med 2021;9(4):277. doi: 10.21037/atm-20-3292 with cancer: american society of clinical oncology clinical practice guideline update 2014. J Clin Oncol 2015;33:654-6.

28. Mulder FI, Candeloro M, Kamphuisen PW, et al. The Khorana score for prediction of venous thromboembolism in cancer patients: a systematic review and meta-analysis. Haematologica 2019;104:1277-87.

29. Imberti D, Benedetti R. Primary prophylaxis of VTE in cancer outpatients. Thromb Res 2016;140 Suppl 1:S103-8.

30. van Es N, Ventresca M, Zhou Q, et al. The Khorana Score for the Prediction of Venous Thromboembolism in Patients with Solid Cancer: An Individual Patient Data Meta-Analysis. Blood 2017;130:627.

31. Lim SH, Woo SY, Kim S, et al. Cross-sectional Study of Patients with Diffuse Large B-Cell Lymphoma: Assessing the Effect of Host Status, Tumor Burden, and Inflammatory Activity on Venous Thromboembolism. Cancer Res Treat 2016;48:312-21.

32. Haas SK, Freund M, Heigener D, et al. Low-molecularweight heparin versus placebo for the prevention of venous thromboembolism in metastatic breast cancer or stage III/IV lung cancer. Clin Appl Thromb Hemost 2012;18:159-65.

33. van Es N, Ventresca M, Di Nisio M, et al. The Khorana score for prediction of venous thromboembolism in cancer patients: An individual patient data meta-analysis. J Thromb Haemost 2020;18:1940-51.

34. Lee YG, Lee E, Kim I, et al. Cisplatin-Based Chemotherapy Is a Strong Risk Factor for Thromboembolic Events in Small-Cell Lung Cancer. Cancer Res Treat 2015;47:670-5. 


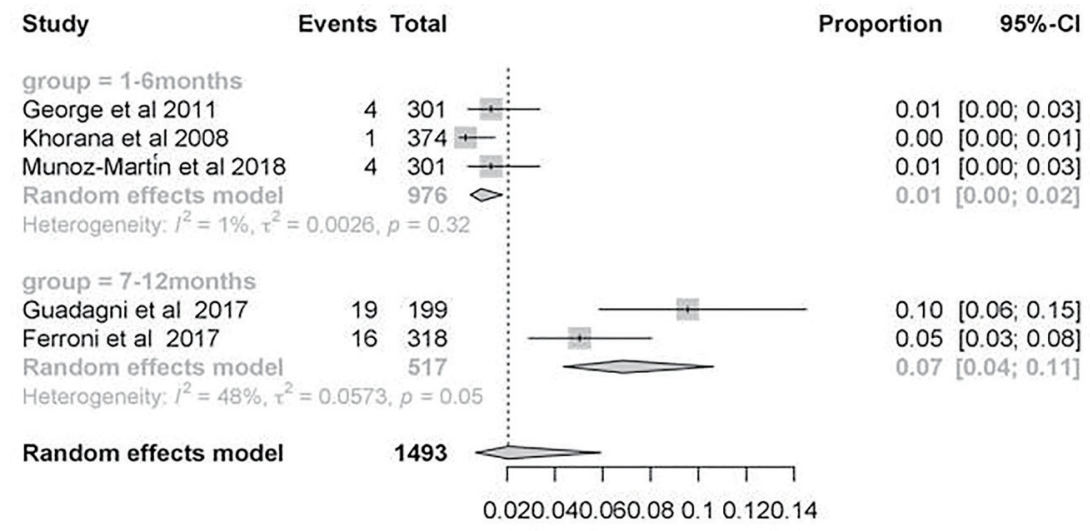

Figure S1 The incidence of different follow-up times in cancer patients at low risk.

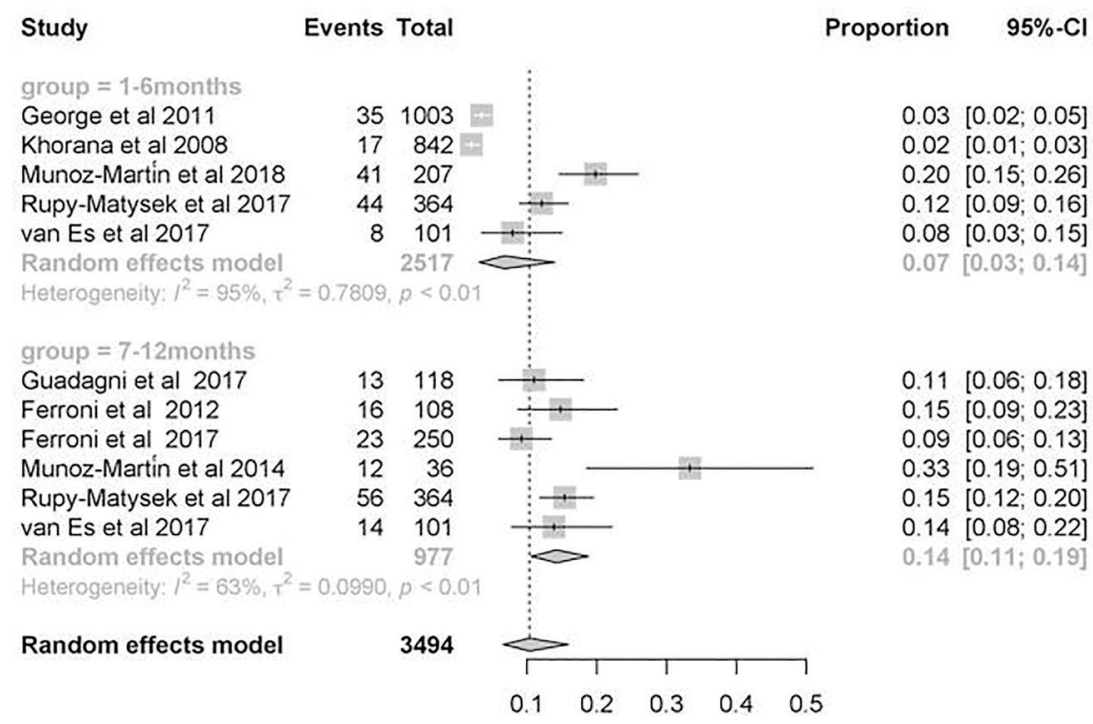

Figure $\mathbf{S} 2$ The incidence of different follow-up times in cancer patients at intermediate risk.

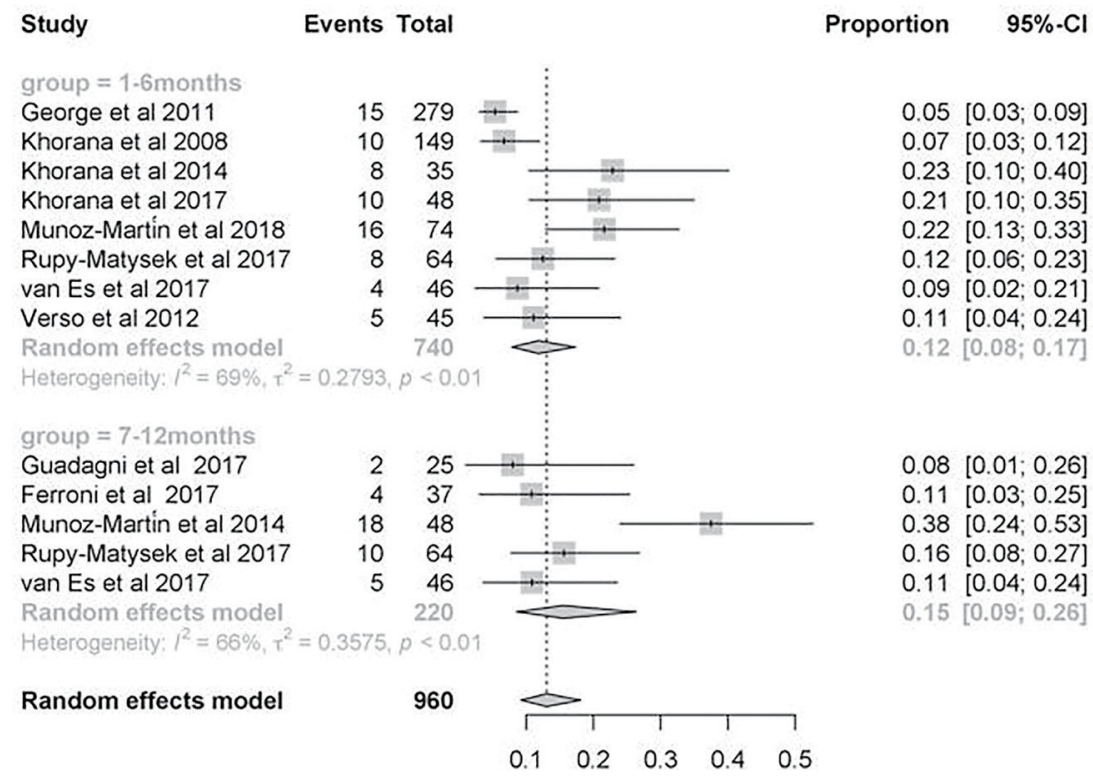

Figure S3 The incidence of different follow-up times in cancer patients at high risk. 
Table S1 Khorana score

\begin{tabular}{lc}
\hline Patient characteristic & Risk score \\
\hline Site of cancer & 2 \\
Very high risk (brain, stomach, pancreas) & 1 \\
High risk (lung, lymphoma, gynecologic, bladder, testicular, myeloma, kidney) & 1 \\
Prechemotherapy platelet count $350 \times 10^{9} / \mathrm{L}$ or more & 1 \\
Hemoglobin level less than $10 \mathrm{~g} / \mathrm{L}$ or use of red blood cell growth factors & 1 \\
Prechemotherapy leukocyte count more than $11 \times 10^{9} / \mathrm{L}$ & 1 \\
BMI $35 \mathrm{~kg} / \mathrm{m}^{2}$ or more & \\
\hline
\end{tabular}

Table S2 Modified Newcastle-Ottawa risk of bias scoring guide

1. Study representativeness:

1 point: prospective study with adequately described inclusion and exclusion criteria

0 point: retrospective study with not adequately described criteria or unclear selection

2. Applicability of Khorana score:

1 point: Khorana score determined for most of the population (>95\%)

0 point: Khorana score could not be calculated for $>5 \%$

3. Outcome measurement:

1 point: blind measurement by an independent assessor.

0 point: no blind measurement or not described

4. Adequacy of follow up of cohorts:

1 point: loss to follow-up was $<5 \%$

0 point: loss to follow-up was not described

5. Applicability outcome:

1 point: LEDVT, UEDVT, PE as outcome

0 point: superficial or abdominal thrombosis included or unclear which types of VTE were included

Studies were judged to be of low risk of bias ( $\geq 2$ points) or high risk of bias (<2 points). LEDVT, lower-extremity deep-vein thrombosis; UEDVT, upper-extremity deep-vein thrombosis; VTE, venous thromboembolism; PE, pulmonary embolism. 